\title{
Optofluidic dye lasers
}

\author{
Zhenyu Li $\cdot$ Demetri Psaltis
}

Received: 1 March 2007 / Accepted: 30 July 2007/Published online: 14 September 2007

(C) Springer-Verlag 2007

\begin{abstract}
Optofluidic dye lasers are microfabricated liquid dye lasers enabled by the microfluidics technology. The integration of dye lasers with microfluidics not only facilitates the implementation of complete "lab-on-a-chip" systems, but also allows the dynamical control of the laser properties which is not achievable with solid-state optical components. We review the recent demonstrations of onchip liquid dye lasers and some of the pre-microfluidics era microscopic dye lasers which are also amenable to microfluidic implementation. Potential applications and future directions are discussed.
\end{abstract}

Keywords Optofluidics - Optofluidic dye lasers .

Microfluidics · Lab-on-a-chip

\section{Introduction}

Optofluidic dye lasers refer to a class of liquid dye lasers, usually on a microfabricated device, in which the liquid medium, besides providing the optical gain, plays a crucial part in determining the optical properties of the laser light such as the lasing wavelength, the spatial mode and tunability. The emergence of optofluidic dye lasers is largely enabled by the recent advances in microfabrication and microfluidics technologies (Xia and Whitesides et al. 1998; Unger et al. 2000). With micromachining and soft lithography techniques, micron-scale fluidic channels can be routinely made in silicon, glass, polymer and elastomer

\section{Z. Li $(\bowtie) \cdot$ D. Psaltis}

Department of Electrical Engineering,

California Institute of Technology,

Pasadena, CA 91125, USA

e-mail: zhenyu@caltech.edu materials. Highly automated microfluidic manipulation and large-scale integration also became possible after the development of soft elastomer-based on-chip mechanical valves and pumps (Unger et al. 2000; Thorsen et al. 2002; Hong and Quake 2003). The advantages of miniaturizing liquid dye lasers on a microfluidic device are threefold. First, microfabricated liquid dye lasers can eliminate many undesirable features of conventional bulk dye lasers which have hindered their widespread use. As is well known, conventional dye lasers have the reputation of "user unfriendly" due to their bulky and complex liquid-handling systems and hazards of toxic dyes. Therefore, in many applications, conventional dye lasers have been replaced by various solid-state laser systems. Microfabrication can dramatically reduce the sizes and complexity of both the optical and the liquid-handling systems. In addition, the extremely small consumption of dye solutions in microfabricated devices not only reduces the cost, but also, combined with the sealed structure, significantly improves the safety and ease of use of dye laser systems. Second, the integration of microfluidics with the adaptive nature of liquid gain medium enables unique optical performances that are not obtainable within solid-state materials (Psaltis et al. 2006). For example, the lasing wavelength can be easily changed by mixing two different solvents to modify the refractive index of the dye solution, or by simply switching from one dye molecule to another ( $\mathrm{Li}$ et al. 2006a, b). The mixing, switching and transport of liquids can be achieved in a highly automated and integrated fashion on a microfluidic device, which is impossible with bulk liquid handing systems. For more comprehensive discussions of the general idea of optofluides, the readers are referred to Psaltis et al. (2006) and Monat et al. (2007). Third and probably the most important feature of the optofluidic dye lasers is their ability to integrate with other 
optical and microfluidic functions to build complete "labon-a-chip" systems. This represents an important step toward fully functional and portable devices for medical diagnostics, environmental monitoring, forensic and bioterrorism detection (Erickson and Li 2004).

The small dimensions of the optofluidic dye lasers are generally achieved using microfabrication techniques. However, there existed micron-sized liquid dye lasers long before the advent of microfluidics which also satisfy our definition of optofluidic dye lasers, such as microdroplet dye lasers (Qian et al. 1986), microcapillary dye lasers (Ippen et al. 1971), and evanescent gain microsphere (An 2001; An and Moon 2003) and fiber lasers (Pendock et al. 1992) immersed in dye solutions. Here, we review both the recent efforts of building on-chip microfluidic dye lasers and some of the pre-microfluidics era dye lasers with micron-scale dimensions, which are also amenable to microfluidic implementations and have potentially interesting applications.

This review is organized as follows. First, in Sect. 2, we briefly discuss the physics of liquid dye lasers including the optical properties of laser dyes, a rate equation model and a simple pump threshold analysis. In Sect. 3, we present some of the pre-microfluidics era microscopic dye lasers and their potential microfluidic implementations. Then, in Sect. 4, we review the recent demonstrations of on-chip microfluidic dye lasers. Finally, we point out the potential applications and future directions in Sect. 5.

\section{Characteristics of liquid dye lasers}

Liquid dye lasers use a solution of organic dye molecules in a solvent, such as an alcohol or water, as the optical gain medium. The ultrawide wavelength tuning range and the ability to generate both narrow linewidth continuous wave (CW) output and ultra short ( $<100 \mathrm{fs})$ pulses make dye lasers an ideal coherent source for spectroscopy (Schafer 1990). The unique features of dye lasers are directly linked to the molecular structures and the photophysical properties of organic dye molecules. Therefore, we will start with the discussion of the properties of laser dyes. On the basis of these properties, we will build a rate equation model for dye lasers and use it to study the pump threshold of the laser action. No attempt is made to give a comprehensive review on conventional dye lasers since a number of excellent review articles (Sorokin et al. 1968; Snavely 1969; Shank 1975; Peterson 1979; Johnston Jr 1987) and books (Schafer 1990; Duarte and Hillman 1990) are already available. Another very useful resource is a collection of original papers on dye lasers, which has been published in the SPIE Milestone Series (Duarte 1992). Textbooks on semiconductor lasers (Coldren and Corzine
1995) and integrated optics (Hunsperger 2002) are also good references for the study of optofluidic dye lasers due to their similar sizes, structures and gain properties.

\subsection{Photophysical properties of dye molecules}

Laser dyes are complex organic molecules containing long chains of conjugated double bonds (i.e., alternating single and double carbon bonds, $=\mathrm{C}-\mathrm{C}=\mathrm{C}-$ ). The large molecular size and the relatively free moving $\pi$-electrons in the conjugated double bonds give rise to the large dipole moments of dye molecules (or equivalently, oscillator strengths). The complex molecular structure also leads to many vibrational and rotational levels within a single electronic state. Therefore, laser dyes often have strong and wide absorption bands in the UV and visible region as shown in Fig. 1 (Peterson et al. 1971). The emission spectra of dye molecules are Stokes-shifted to longer wavelength and form almost mirror images of the absorption bands due to the so-called Frank-Condon principle (Svelto 1998). This is a rather fortunate feature because the laser emission will generally not be strongly absorbed by the dye itself. In the following, we will briefly discuss the energy levels and the transition processes involved in laser operation, with a special focus on the effects of the dark triplet states. For more complete discussions of the photophysical and photochemical properties of laser dyes including the environmental effects, the readers are referred to Schafer (1990).

Figure 2 shows the typical energy levels of a dye molecule. $S_{0}$ and $S_{1}$ are the ground and the first excited singlet

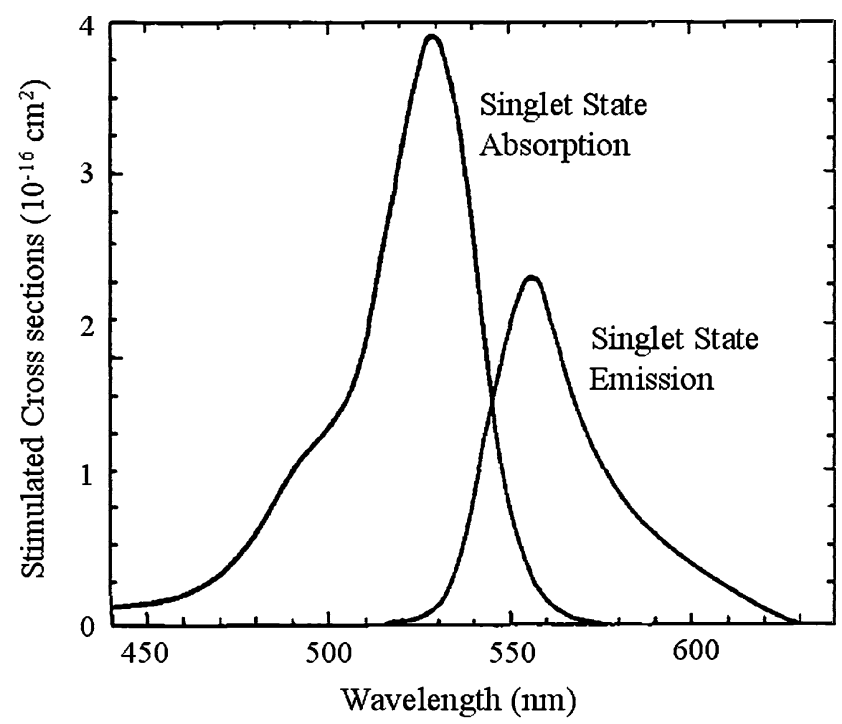

Fig. 1 Stimulated singlet state absorption and emission cross sections for Rhodamine $6 \mathrm{G}$ in ethanol. Reprint with permission from Peterson et al. (1971) 
electronic states. $T_{1}$ and $T_{2}$ are the first and second excited triplet states. In a singlet state, the spin of the excited electron is anti-parallel to the spin of the remaining molecule and the total spin quantum number is $S=0$, whereas in a triplet state the two spins are parallel and $S=1$. Therefore, singlet-singlet and triplet-triplet transitions are allowed, while singlet-triplet transitions are forbidden because of the selection rule $\Delta S=0$. As mentioned earlier, each electronic state is further split into many vibrational and rotational sublevels due to the complex dye molecular structure. In addition, the effective homogenous linebroadening mechanism due to the collisions with solvent molecules smears these sublevels into unresolved overlapping bands. The homogenous broadened spectra of dye molecules enable very efficient channeling of the pump energy into narrow band laser emissions (Johnston Jr 1987).

It is important to realize that the dye laser is a classical four-level system (if neglect the triplet states) where the four energy levels are marked 0-3 in Fig. 2. Under optical excitation, dye molecules are pumped from the bottom of the ground state $S_{0}$ (ground level 0 ) to some vibrationalrotational sublevels in the first singlet state $S_{1}$ (intermediate pump level 3). The dye molecules in level 3 quickly relax to the bottom of $S_{1}$ (upper laser level 2). This nonradiative decay process happens on the time scale of a few picoseconds or less. The energy lost in this process contributes to the heating of the solvent. From the upper laser level 2, dye molecules can undergo either spontaneous emission or stimulated emission to some vibrational-rotational sublevels in $\mathrm{S}_{0}$ (lower laser level 1). The nonradiative decay time from the lower laser level 1 to the bottom of the

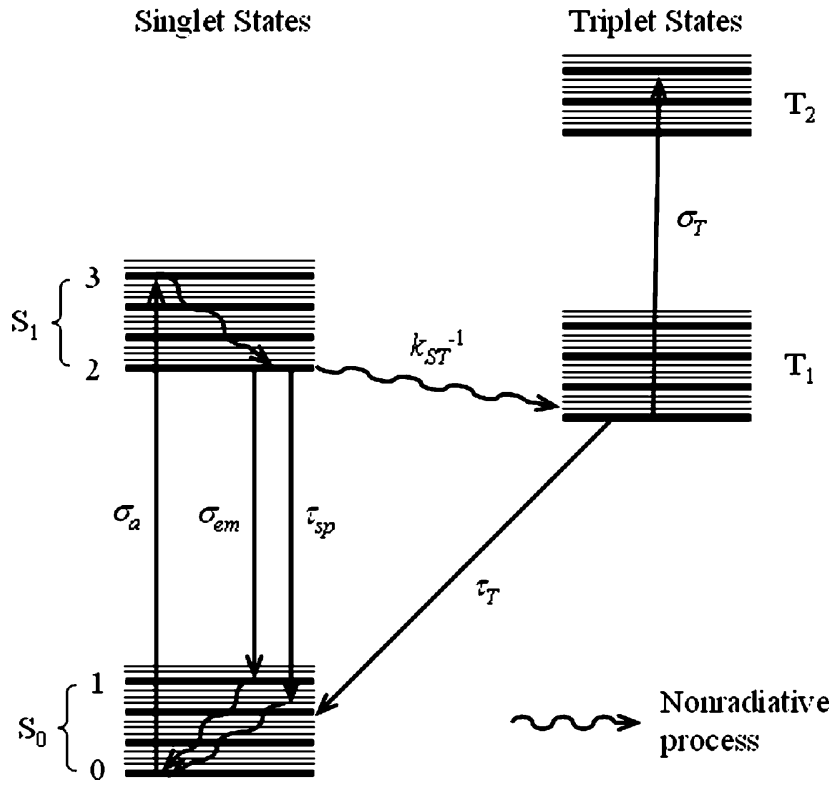

Fig. 2 Typical energy levels of a dye molecule. Each transition is labeled by its corresponding cross section or lifetime ground state $S_{0}$ (ground level 0 ) is also a few picoseconds. Due to the extremely fast nonradiative decay processes, the populations of the lower laser level $n_{1}$ and the intermediate pump level $n_{3}$ are negligibly small. Therefore, dye lasers can reach threshold at very small population inversions, with typical $n_{2} / n$ of only 0.01 or less (Johnson Jr 1987). However, due to the short upper state lifetimes (a few nanoseconds), dye lasers still need high pump intensities to reach threshold (see Eq. 7 in Sect. 2.3).

The real situations are further complicated by the triplet state effects. The excited molecules at the upper laser level 2 undergo not only radiative transitions to $S_{0}$ but also nonradiative intersystem crossing to the dark triplet state $T_{1}$. As mentioned before, this process requires a spin flip of the excited electron and thus is spin-forbidden. This is the reason that intersystem cross has a relative slow transition rate $\left(1 / k_{\mathrm{ST}} \sim 100 \mathrm{~ns}\right)$ and the triplet state lifetime is long $\left(\tau_{\mathrm{T}} \sim 100 \mathrm{~ns}\right)$. The metastable triplet state has two detrimental effects on laser oscillation. First, the long-lived triplet state $T_{1}$ traps the dye molecules and decreases the available population inversion. Second, the absorption from the first triplet state $T_{1}$ to the second triplet state $T_{2}$ tends to overlap with the laser emission and produces extra losses at the laser wavelength. Therefore, short pump pulses, fast circulation of dye solutions and triplet state quenchers are often utilized to minimize the triplet state influences in different types of dye lasers. For example, in conventional $\mathrm{CW}$ dye lasers, the dye solutions flow by the excitation region at velocities as high as $10 \mathrm{~m} / \mathrm{s}$ to circumvent the triplet state trapping and thermal distortion problems.

\subsection{Rate equations}

Rate equations describe the time evolution of the energy level populations and the laser cavity mode. Although they do not account for the coherent properties of the laser light, they are very useful for the estimations of the laser threshold and output power.

For dye lasers, three rate equations are needed for describing the laser cavity mode photon flux $\phi\left(=I_{\mathrm{L}} / \mathrm{h} v_{\mathrm{L}}\right)$, the singlet state population density $n_{2}$ and the triplet state population density $n_{\mathrm{T}}$.

Laser cavity mode $\phi$ :

$\frac{\mathrm{d} \phi}{\mathrm{d} t}=c\left[n_{2} \sigma_{\mathrm{em}}\left(\lambda_{\mathrm{L}}\right)-n_{0} \sigma_{\mathrm{a}}\left(\lambda_{\mathrm{L}}\right)-n_{\mathrm{T}} \sigma_{\mathrm{T}}\left(\lambda_{\mathrm{L}}\right)-\frac{\gamma}{L}\right] \phi$

where $\sigma_{i} \mathrm{~s}$ are the stimulated transition cross sections at the laser wavelength $\lambda_{\mathrm{L}}, n_{0}$ is the ground state population density, $\gamma$ is the single pass cavity loss (equals $-\ln \left(R_{1} R_{2}\right) /$ $2+\gamma_{i}$ for Fabry-Perot cavities with internal loss $\gamma_{i}$ ), $L$ is the length of the gain region and $c$ the speed of light. 
First excited singlet state $n_{2}$ :

$\frac{\mathrm{d} n_{2}}{\mathrm{~d} t}=R_{\mathrm{P}}+\left[n_{0} \sigma_{\mathrm{a}}\left(\lambda_{\mathrm{L}}\right)-n_{2} \sigma_{\mathrm{em}}\left(\lambda_{\mathrm{L}}\right)\right] \phi-n_{2} k_{\mathrm{ST}}-\frac{n_{2}}{\tau_{\mathrm{sp}}}$

where $k_{\mathrm{ST}}$ is the intersystem crossing rate, $\tau_{\mathrm{sp}}$ is the spontaneous emission lifetime, and the pump rate per unit volume $R_{\mathrm{P}}$ is given by

$R_{\mathrm{P}}=n_{0} \sigma_{\mathrm{a}}\left(\lambda_{\mathrm{P}}\right) I_{\mathrm{P}} / h v_{\mathrm{P}}$

where $I_{\mathrm{P}}$ is the pump intensity, $\lambda_{\mathrm{P}}$ and $v_{\mathrm{P}}$ are the pump light wavelength and frequency, respectively, and $h$ is Planck's constant.

First excited triplet state $n_{\mathrm{T}}$ :

$\frac{\mathrm{d} n_{T}}{\mathrm{~d} t}=n_{2} k_{\mathrm{ST}}-\frac{n_{\mathrm{T}}}{\tau_{\mathrm{T}}}$

where $\tau_{\mathrm{T}}$ is the triplet state lifetime.

Finally, the conservation of molecules requires

$n=n_{0}+n_{2}+n_{\mathrm{T}}$

where $n$ is the total concentration of dye molecules.

The typical values of the photophysical parameters needed in the rate equations are listed in Table 1 for the most common laser dye, Rhodamine 6G (Duarte and Hillman 1990).

\subsection{Pump threshold analysis}

Our analysis of the pump threshold is similar to those given in Svelto (1998) and Davis (1996). A more thorough treatment can be found in Peterson et al. (1971). In general,

Table 1 Typical values of the photophysical parameters for Rhodamine $6 \mathrm{G}$ (pump wavelength $\lambda_{\mathrm{P}}=532 \mathrm{~nm}$, laser wavelength $\left.\lambda_{\mathrm{L}}=580 \mathrm{~nm}\right)$

\begin{tabular}{ll}
\hline Parameter & Value \\
\hline $\begin{array}{l}\text { Absorption cross section at the } \\
\text { pump wavelength } \sigma_{\mathrm{a}}\left(\lambda_{\mathrm{P}}\right)\left(\mathrm{cm}^{2}\right)\end{array}$ & $3.8 \times 10^{-16}$ \\
$\begin{array}{l}\text { Absorption cross section at the } \\
\quad \text { laser wavelength } \sigma_{\mathrm{a}}\left(\lambda_{\mathrm{L}}\right)\left(\mathrm{cm}^{2}\right)\end{array}$ & $1 \times 10^{-19}$ \\
$\begin{array}{l}\text { Stimulated emission cross } \\
\quad \text { section } \sigma_{\mathrm{em}}\left(\lambda_{\mathrm{L}}\right)\left(\mathrm{cm}^{2}\right)\end{array}$ & $1.2 \times 10^{-16}$ \\
$\begin{array}{l}\text { Triplet state absorption cross } \\
\quad \text { section at the laser wavelength }\end{array}$ & $1 \times 10^{-17}$ \\
$\quad \sigma_{\mathrm{T}}\left(\lambda_{\mathrm{L}}\right)\left(\mathrm{cm}^{2}\right)$ & \\
Spontaneous emission lifetime $\tau_{\mathrm{sp}}(\mathrm{ns})$ & 5 \\
Intersystem crossing time $1 / k_{\mathrm{ST}}(\mathrm{ns})$ & 100 \\
Triplet state lifetime $\tau_{\mathrm{T}}(\mathrm{ns})$ & $100^{\mathrm{a}}$ \\
\hline
\end{tabular}

$\overline{\text { a }}$ Vary from $10^{-7}$ to $10^{-3} \mathrm{~s}$ depending on the solvent and the presence or absence of the triplet quencher the pump threshold can be obtained by numerically solving the rate equations. However, as shown in the following, under certain approximations, analytic expressions can be derived to give reasonable estimates.

\subsubsection{Pulsed operation}

In pulsed operation with pump pulse duration shorter than the intersystem crossing time $1 / k_{\mathrm{ST}}$, we can neglect the triplet state effects. Also we assume that the self-absorption at the laser wavelength is small. In this case, the threshold population inversion density is determined only by the cavity loss (Svelto 1998)

$n_{2 \mathrm{th}}=\frac{\gamma}{\sigma_{\mathrm{em}}\left(\lambda_{\mathrm{L}}\right) L}$

Therefore, the threshold pump intensity needed to achieve this upper state population is

$I_{\mathrm{th}}=n_{2 \mathrm{th}} h v_{\mathrm{P}} d / \tau_{\mathrm{sp}}=\frac{\gamma h v_{\mathrm{P}} d}{\sigma_{\mathrm{em}}\left(\lambda_{\mathrm{L}}\right) \tau_{\mathrm{sp}} L}$

where $d$ is the penetration depth of the pump light into the dye solution, given by $1 / n_{0} \sigma_{\mathrm{a}}\left(\lambda_{\mathrm{P}}\right)$. For simplicity we do not consider the spatial dependence of the pump rate and the specific pumping geometry. The readers can find the more accurate treatments in most laser textbooks (Svelto 1998; Silfvast 2004).

\subsubsection{CW operation}

In $\mathrm{CW}$ operation, the triplet state population $n_{\mathrm{T}}$ at equilibrium (steady state) can be obtained from Eq. 4,

$n_{\mathrm{T}}=n_{2} k_{\mathrm{ST}} \tau_{\mathrm{T}}$

The threshold gain equals the total loss and, from Eq. 1, is given by

$g_{\text {th }}=n_{2} \sigma_{\mathrm{em}}=n_{0} \sigma_{\mathrm{a}}+n_{\mathrm{T}} \sigma_{\mathrm{T}}+\gamma / L$

where all the cross sections are evaluated at the laser wavelength. With the help of the population conservation relation (5), we get the threshold population inversion

$n_{2 \mathrm{th}}=\frac{n \sigma_{\mathrm{a}}+\gamma / L}{\sigma_{\mathrm{em}}+\sigma_{\mathrm{a}}\left(1+k_{\mathrm{ST}} \tau_{\mathrm{T}}\right)-\sigma_{\mathrm{T}} k_{\mathrm{ST}} \tau_{\mathrm{T}}}$

In all practical $\mathrm{CW}$ dye lasers, the excited dye molecules are quickly removed from the excitation region and a triplet state quencher is often added to the solution. These two methods effectively reduce the triplet state lifetime by several orders of magnitude. Again for many efficient laser 
dyes, the self-absorption cross section is negligibly small. Consequently, we can set $k_{\mathrm{ST}} \tau_{\mathrm{T}} \sim 0$ and $\sigma_{\mathrm{a}} \sim 0$ in Eq. 10, which reduces it to the same expression as Eq. 6. Therefore, we can use expression (7) to roughly estimate the pump threshold for both pulsed and CW dye lasers. For typical values $\sigma_{\mathrm{em}}=1 \times 10^{-16} \mathrm{~cm}^{2}, \tau_{\mathrm{sp}}=5 \mathrm{~ns}, d=1 \mathrm{~mm}$, $L=10 \mathrm{~mm}, \gamma=0.01, v_{\mathrm{P}}=5.6 \times 10^{14} \mathrm{~Hz}(530 \mathrm{~nm})$, the calculated threshold pump intensity is approximately $740 \mathrm{~W} / \mathrm{cm}^{2}$, the corresponding population inversion $n_{2}$ is about $1 \times 10^{16} \mathrm{~cm}^{-3}$ and the threshold gain coefficient is about $1 \mathrm{~cm}^{-1}$. In practical systems, the threshold intensity is usually much higher, about $50 \mathrm{~kW} / \mathrm{cm}^{2}$, due to the triplet state effects, self-absorption, thermal distortions and higher cavity losses.

\section{Pre-microfluidics era dye lasers}

Liquid dye lasers with micron-scale dimensions have been demonstrated long before the birth of the microfluidics technology. This is because, unlike solid-state lasers for which microfabrication processes are usually needed to define the miniaturized optical structures, the flexibility of liquid gain medium allows the formation of micron-scale optical cavities in several straightforward ways without using any microfabrication technique, such as microdroplet formation, microcapillary filling and immersion of small solid transparent optical structures in dye solutions. Here, we divide the pre-microfluidics era optofluidic dye lasers into two categories: microcavity dye lasers and waveguide dye lasers, in which the microcavity dye lasers are further divided into three subcategories on the basis of the cavity type: microdroplets, evanescent gain microcavities and Fabry-Perot-type microcavities. We give representative examples in each category and discuss their possible implementations using the microfluidics technology.

\subsection{Microcavity dye lasers}

Optical microcavities are resonant structures that confine light in small volumes. A major application of microcavities is to build compact and efficient laser sources. Liquid dye solutions are among the first material systems used to study the laser action and other optical processes in microcavities (Chang and Campillo 1996; Vahala 2003). First, we will discuss dye lasers in surface tension-induced microdroplet cavities.

\subsubsection{Microdroplet dye lasers}

Microdroplets, with their near atomically smooth surfaces and small sizes, offer an ideal optical cavity for making low threshold and compact dye lasers. In such microspherical cavities, light is confined by continuous total internal reflections at the liquid-air interface, and forms the so-called whispering gallery modes (WGMs). Dye lasing has been demonstrated in free falling droplets (Tzeng et al. 1984; Qian et al. 1986), pendant droplets (Hansch 2005) and levitated droplets (Tona and Kimura 2000; Azzouz et al. 2006). Although the observation of dye lasing in droplets dates back to the 1970s (Hansch 2005), the first reported microdroplet dye laser in the literature was by Chang's group (Tzeng et al. 1984; Qian et al. 1986). In this work, dye solution droplets were generated by a vibrating orifice, in which a cylindrical liquid jet passing through the orifice is induced to break up into equal-sized droplets by a mechanical vibration of the orifice with the proper frequency and amplitude (Tzeng et al. 1984; Lin et al. 1990). The main experimental results for $60 \mu \mathrm{m}$ diameter ethanol droplets containing Rhodamine $6 \mathrm{G}$ are shown in Fig. 3. The droplets were pumped by $10 \mathrm{~ns}$ laser pulses at $532 \mathrm{~nm}$. Lasing occurs only in the longer wavelength side of the emission spectrum due to the high self-absorption at shorter wavelengths (Fig. 3, right panel). The laser emission is confined near the surfaces of the droplets which is characteristic for WGMs (Fig. 3b). Pump threshold of $\sim 35 \mathrm{~W} / \mathrm{cm}^{2}$, which was three orders of magnitude lower than those of bulk dye lasers, was achieved (Tzeng et al. 1984). The cavity $Q$ factor of such droplets can be as high as $10^{8}$ for low dye concentrations $\left(<10^{-5} \mathrm{M}\right)$ (Eversole et al. 1993). The characteristics of such droplet dye lasers were comprehensively studied and reviewed (Lin et al. 1986, 1992a; Chang and Campillo 1996).

Besides the laser applications, microdroplets have also been used to study cavity-induced spontaneous emission modification (Campillo et al. 1991; Lin et al. 1992b; Barnes et al. 1992), single molecule fluorescence (Barnes et al. 1993, 1996; Lermer et al. 1997), and many nonlinear optical effects such as stimulated Raman scattering, multiorder Stokes emission and stimulated Brillouin scattering (Chang and Campillo 1996, Chaps. 5-7, and references therein).

Although microdroplet cavities hold great potentials for laser applications, biochemical sensing, single molecule studies, nonlinear optics and cavity quantum electrodynamics (cQED), the complexity and inconvenience of the setup, and the short evaporative lifetimes of the droplets have limited them mainly in a few research laboratories. Interestingly, several recently demonstrated on-chip microdroplet generators may change the situation (Thorsen et al. 2001; Anna et al. 2003). With such devices, monodispersed droplets over a wide range of sizes $(1-500 \mu \mathrm{m})$ can be automatically generated in a microfluidic channel at very high speed. Furthermore, the sizes and shapes of individual droplets and the distances between droplets can 
Fig. 3 Microdroplet dye laser. Left Photographs of laser emission from the droplet stream. a The upper portion of the stream (close to the orifice) showing the breaking of the dye solution and the development of highly distorted droplets. b The lower portion of the stream showing the transition from oscillating spheroids to monodisperesed spherical droplets. c A photograph of combined laser emission (red) and elastic scattering (green). Right d Schematic

representation of the absorption and emission spectra of emission spectrum of the $60 \mu \mathrm{m}$ diameter droplets for region $B$. Reprinted with permission from Qian et al. (1986) Rhodamine $6 \mathrm{G}$ in ethanol. e The
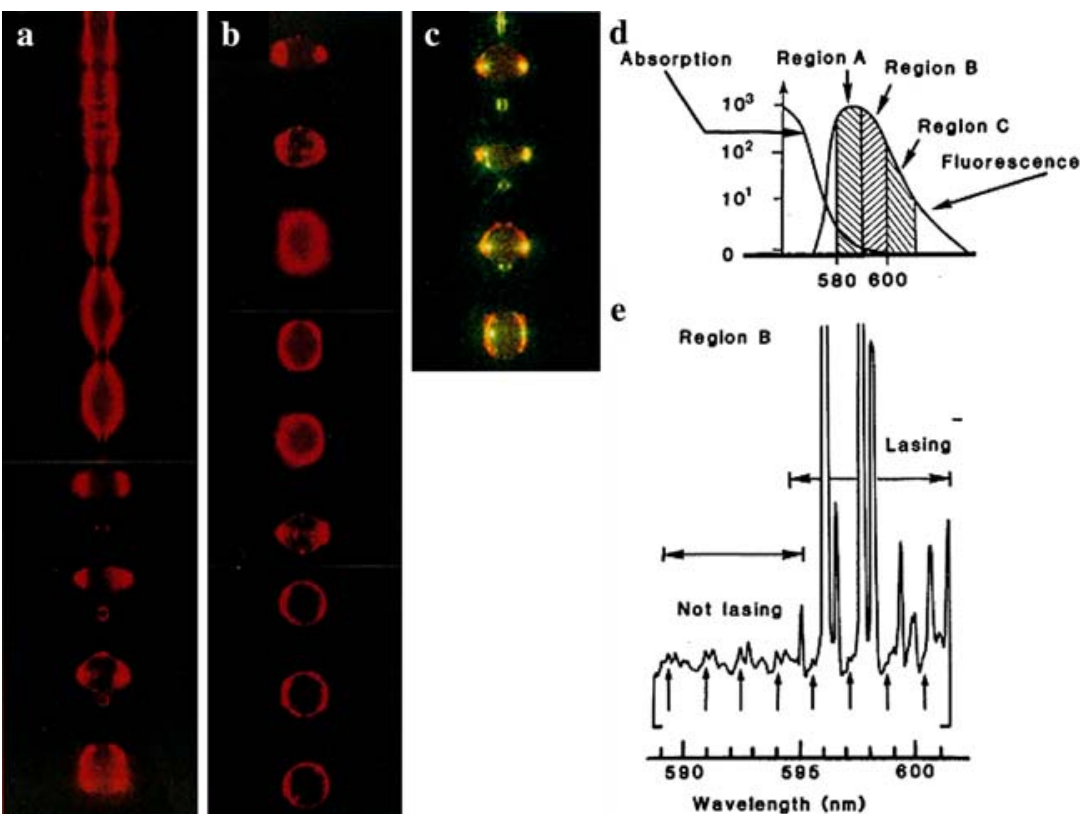

be accurately controlled by changing the liquid flow rate and the dimensions of the microfluidic channels. This will enable more systematic studies of new phenomena such as the chaotic behaviors and the directional emissions in nonspherical droplets (Mekis et al. 1995; Nockel et al. 1996) and the coupling effects among many droplet cavities which are difficult, if not impossible, to study with conventional bulk droplet generators. Also, as mentioned above, this should provide an on-chip platform for biochemical sensing, single molecule studies, liquid-based nonlinear optics and quantum optics. Finally, the ultrahigh $Q$ s and small mode volumes make these cavities promising candidates for LED pumped on-chip laser sources ( $\mathrm{Li}$ and Psaltis 2007).

\subsubsection{Evanescent gain microcavity dye lasers}

When the evanescent tail of an optical wave is immersed in a liquid gain medium, it can extract energy and lead to optical amplification. Several groups have demonstrated microcavity liquid dye lasers on the basis of such evanescent wave coupled gain, typically by immersing a WGM resonator such as a microsphere or a microcylinder into a dye solution. Fujiwara and Sasaki (1999) demonstrated laser action using silica microspheres dispersed in an aqueous solution of Rhodamine B. They used the fundamental pulses of a $Q$-switched Nd:YAG laser at $1,064 \mathrm{~nm}$ to optically trap a single microsphere, and the second harmonic pulses at $532 \mathrm{~nm}$ to pump the surrounding dye molecules. The combination of optical trapping and laser spectroscopy allowed the authors to freely manipulate individual lasing microspheres in three dimensions and measure the lasing spectra. The lasing threshold was determined to be $7 \mathrm{~mW}$ ( $30 \mathrm{~ns}$ pulses, $10 \mathrm{kHz}$ repetition rate) for a $28-\mu \mathrm{m}$ diameter microsphere in a 10-mM Rhodamine B solution. The measured spectra agreed well with the Mie scattering theory prediction. Figure 4 shows another evanescent gain dye laser with an ultrahigh $Q$ cylindrical microcavity, which is formed by a fused silica optical fiber located in a capillary tube filled with a low index $2 \mathrm{mM}$ Rhodamine 6G solution (Moon et al. 2000b). Without the center cylindrical cavity, the system showed conventional Fabry-Perot-type lasing due to the front and back interfaces [Fig. 4b(1)]. However, when an optical fiber (the cylindrical microcavity) of $125 \mu \mathrm{m}$ diameter was inserted into the same capillary, WGM laser oscillation was achieved with a low-threshold pump energy of $\sim 200 \mu \mathrm{J}$ [Fig. $4 \mathrm{~b}(2)$, c]. The $Q$ factors of these microcavities was inferred from a four-level laser model to be $3 \times 10^{7}$ for the TM modes of radial order $l=2$. Even higher $Q$ of $10^{9}$ was achieved when a silica microsphere was used and the dye concentration was lowered to $0.05 \mathrm{mM}$ (Choi et al. 2001; An and Moon 2003). Later, this structure was used to demonstrate laser oscillation with colloidal semiconductor $\mathrm{CdSe} / \mathrm{ZnS}$ quantum rods as gain medium (Kazes et al. 2002). Microring waveguide mode dye lasers were also demonstrated using the structure shown in Fig. $4 b(1)$, in which the high index capillary wall forms the microring cavity (Moon et al. 2000a; Shopova et al. 2007).

Thus far, all the demonstrated evanescent gain microcavity dye lasers are based on isolated optical components such as chemically synthesized microspheres, melting induced silica microspheres, optical fibers or capillary tubes. However, there is no reason that such lasers cannot 
Fig. 4 a Cylindrical evanescent gain microcavity dye laser. b Schematics and microscope images of (1) radial mode lasing and (2) WGM lasing. c Typical spectra of WGM lasing with the central optical fiber inserted. Inset Typical single shot spectra from the radial mode lasing without the central fiber. Reprinted with permission from Moon et al. (2000b)

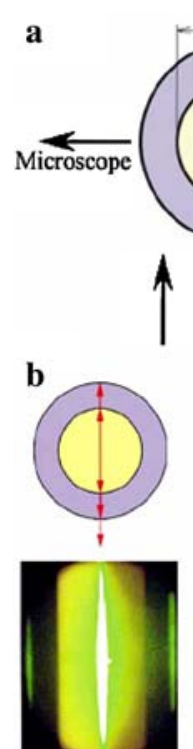

(1)

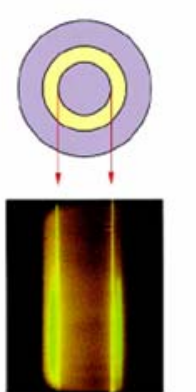

(2) be made on a microfabricated device integrated with microfluidic liquid handling. In fact, the evanescent gain structure is quite favorable for rapid gain medium replenishment and sensing applications (Yang and Guo 2006).

\subsubsection{Fabry-Perot-type microcavity dye lasers}

Another way to build an optical microcavity is to shrink the mirror spacing of a Fabry-Perot resonator. When the mirror spacing is sufficiently small, the free spectral range (FSR) of the cavity becomes larger than the emission bandwidth, and leads to single longitudinal mode operation. The first optical microcavity experiments used dye molecules between high reflectivity dielectric Bragg reflectors in the Fabry-Perot configuration (De Martini and Jacobovitz 1988; Suzuki et al. 1991). The main purpose of such experiments was to study the spontaneous emission modification through the Purcell effect (Purcell 1946). For example, Fig. 5 shows the emission behavior of a dielectric mirror-based Fabry-Perot microcavity dye laser (Yokoyama et al. 1991; Yokoyama 1992). The cavity length was accurately defined by $\mathrm{Ti}$ spacers deposited on one of the mirrors. The space between the mirrors was filled with dye solution by capillary forces. When the mirror spacing is half the wavelength $(d=\lambda / 2)$, the fraction of spontaneous emission coupled into the cavity mode $(\beta)$ becomes large compared to that of a conventional macroscopic laser. This not only increases the spontaneous emission rate but also makes it highly directional. In principle, the traditional laser threshold can disappear when $\beta$ approaches 1, leading to the so-called thresholdless laser oscillation (Yokoyama and Brorson 1989; De Martini and Jacobovitz 1988;
Yokoyama 1992). The $\beta$ factor of $\sim 0.2$ was achieved in this experiment for the $d=\lambda / 2$ cavity. As shown in the output versus input curves (Fig. 5b), the laser threshold became quite fuzzy for the short cavities, while for the long cavity it was clear. Also the spectra of the spontaneous emission and the laser action were very similar for the short cavity laser, while for the long cavity, the spectra changed dramatically above the threshold (Fig. 5c).

Microcavities have many attractive features besides the ultralow laser threshold, such as the ultrafast response time, nonclassical photon statistics and the ability to generate well defined triggered single photons (Yokoyama 1992; Vahala 2003). Therefore, their implementation on a dynamical optofluidic device should be of great interest for both fundamental studies and technical applications. As we will show in Sect. 4, the recent demonstrations of on-chip optofluidic dye lasers represent a big step closer toward an on-chip tunable microcavity dye laser system for studying the fundamental light-matter interactions.

\subsection{Waveguide dye lasers}

Similar to microcavity dye lasers, liquid core or liquid cladding waveguide dye lasers can be made with capillary tubes and optical fibers. Ippen and coworkers built liquidcore waveguide dye lasers by filling small diameter $(\sim 10 \mu \mathrm{m})$ glass capillary tubes $(n=1.48)$ with high refractive index dye solutions in benzylalcohol $(n=1.53)$ (Ippen et al. 1971). They used this structure to study the photobleaching lifetimes of various laser dyes under $\mathrm{CW}$ laser pump. It was found that Rhodamine $6 \mathrm{G}$ molecules in ethanol solution, when pumped at intensities required for 
Fig. 5 a Schematic of a FabryPerot microcavity dye laser. b Light output vs. input curves. For short cavity lasers, the laser threshold became fuzzy while a clear threshold appeared for the $d=500 \lambda$ cavity. $\mathbf{c}$ Spontaneous and laser emission spectra. For the $d=\lambda / 2$ cavity, the two spectra were very similar. However, for the $d=500 \lambda$ cavity, a dramatic spectral narrowing occurred above threshold. Reprinted with permission from Yokoyama (1992)
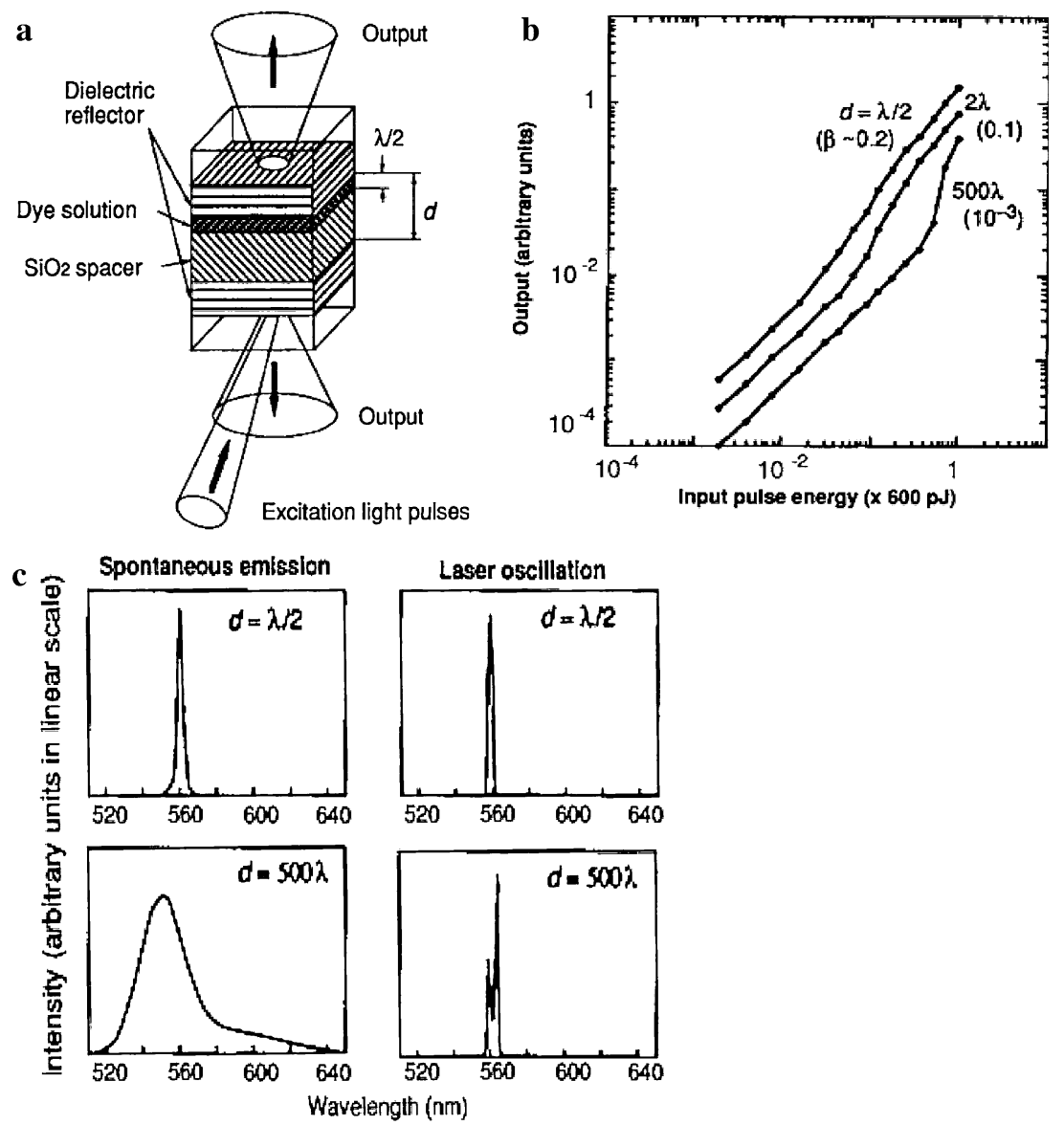

laser action $\left(\sim 100 \mathrm{~kW} / \mathrm{cm}^{2}\right)$, have a usable lifetime of only $40 \mathrm{~ms}$. This work established that the short photobleaching lifetimes of most dye molecules strongly limit the usefulness of any nonflowing dye laser structures. Later, a thin film waveguide dye laser, in which the dye solution lying external to the waveguide core was excited via evanescent fields, was built to circumvent this problem because the bleached dye molecules can now be replenished by diffusion or liquid flow across the waveguide surface (Ippen and Shank 1972).

Zeidler built, to our knowledge, the first integrated optical waveguide dye laser by evaporating dielectric channel structures on a glass substrate and bonding a second glass plate on top (Zeidler 1971). A $5 \mathrm{~mm}$ long liquid core waveguide with a cross section of $10 \mu \mathrm{m}$ by $300 \mu \mathrm{m}$ was made. The waveguide core was filled with the dye solution of Rhodamine B dissolved in benzylalcohol by capillary forces. The dye solution was then pumped by $30 \mathrm{~ns}$ Nd:YAG laser pulses at $532 \mathrm{~nm}$ and the laser oscillation was achieved with only the reflections from the dye solution/air interfaces at the two ends of the waveguide. Recently, with the invention of photonic crystal fibers and microstructured fibers (Russell
2003), waveguide dye lasers can be made with even smaller core dimensions and longer interaction lengths, which provides a convenient platform for sensing and spectroscopy applications (Vasdekis et al. 2007).

When a tapered optical fiber is immersed in a low refractive index dye solution and a pump laser beam is launched into the taper, an evanescent gain waveguide dye laser is achieved (Pendock et al. 1992 and Pendock et al. 1993). The use of a tapered fiber waveguide allows simple and efficient coupling of the pump beam, as well as the collection of the laser output. The small size of the taper also gives rise to a large modal gain coefficient due to the large evanescent tail inside the gain medium, and meanwhile ensures single transverse mode operation. Periasamy and Bor used a similar evanescent gain structure with large diameter $(50 \mu \mathrm{m})$ uncladded quartz fibers (Periasamy and Bor 1981). However, instead of using longitudinal pumping, they used the interference pattern of two pulsed $\mathrm{N}_{2}$ laser beams at $337.1 \mathrm{~nm}$ to transversely pump the dye solution and achieved gain modulated distributed feedback (DFB) lasing. The laser wavelength can be tuned by changing the angle between the two interfering beams. A small signal gain of 430 (gain coefficient $\sim 3 \mathrm{~cm}^{-1}$ ) at 
$600 \mathrm{~nm}$ has been measured in a $2 \mathrm{~cm}$ long fiber surrounded by an excited Rhodamine 6G solution (Periasamy and Schafer 1981).

Now with the well developed microfluidics technology, it is straightforward to build on-chip waveguide dye lasers by replacing the optical fibers or capillaries with the integrated optical waveguides (Vezenov et al. 2005; Li et al. 2006a, b).

\section{Microfluidics-based dye lasers}

The development of microfluidics in the 1990s has created a powerful toolbox for manipulating liquids at the nanoliter scale or below (Whitesides 2006). An important goal for microfluidics is to achieve complete "lab-on-a-chip" systems, in which optical functions are often indispensable (Chabinyc et al 2001; Verpoorte 2003). Meanwhile, the power of microfluidic control has been recognized for building adaptive and reconfigurable optical systems (Psaltlis et al. 2006). One important example of such systems is the optofluidic dye lasers, in which the adaptive nature of the liquid gain medium and the combination with microfluidic liquid handing can enable unprecedented optical performances and high degree of system integration.

\subsection{Early demonstrations with wide band output}

The first on-chip liquid dye laser on the basis of the modern microfluidics technology was demonstrated by Kristensen's group (Helbo et al. 2003). Figure 6 shows the structure and the emission spectrum of the device. A surface emitting Fabry-Perot cavity with metallic mirrors was fabricated on a SU8 and Pyrex glass hybrid chip by standard microfabrication techniques. The top mirror was made of $5 \mathrm{~nm} \mathrm{Cr}$ and $40 \mathrm{~nm} \mathrm{Au}$, and had a reflectance $\mathrm{R} 1=72 \%$ and a transmittance $\mathrm{T} 1=6 \%$. The bottom mirror was made of $10 \mathrm{~nm} \mathrm{Cr}$ and $150 \mathrm{~nm} \mathrm{Au}$, and had an R2 = 83\% and a $\mathrm{T} 2=0 \%$. A dye solution of $10 \mathrm{mM}$ Rhodamine $6 \mathrm{G}$ in ethanol was pumped through the $10 \mu \mathrm{m}$ deep and $1 \mathrm{~mm}$ wide microfluidic channel between the mirrors at a flow rate of $10 \mu \mathrm{l} / \mathrm{min}$ with a syringe pump. The optical path length between the mirrors was $\sim 20 \mu \mathrm{m}$. The pump light are $5 \mathrm{~ns}$ frequency doubled $\mathrm{Nd}: \mathrm{YAG}$ laser pulses at $532 \mathrm{~nm}$ with a repetition rate of $10 \mathrm{~Hz}$. Laser emission at $570 \mathrm{~nm}$ with a FWHM linewidth of $5.7 \mathrm{~nm}$ was achieved at the pump level of $368 \mathrm{~mW} / \mathrm{cm}^{2}$. The laser threshold was estimated to be $34 \mathrm{~mW} / \mathrm{cm}^{2}$.

Vezenov et al. (2005) fabricated an edge emitting dye laser in poly(dimethylsiloxane) (PDMS) using soft lithography. The laser cavity was based on a liquid-liquid $\left(\mathrm{L}^{2}\right)$ waveguide (Wolfe et al. 2004). Gold mirrors deposited at the ends of the waveguide formed a Fabry-Perot cavity. The $\mathrm{L}^{2}$ waveguide allowed the dynamical control of the waveguide and cavity properties by adjusting the index contrast between the core and cladding liquids. By mixing methanol and DMSO, peak lasing wavelength was tuned from 617 to $634 \mathrm{~nm}$.

A free space ring-cavity-based microfluidic dye laser was fabricated in a photoechable glass, Foturan, by femtosecond laser microprocessing (Cheng et al. 2004). The 3D microprocessing permitted the fabrication of devices with multi-layered structures. An array of two lasers operated simultaneously under one pump light was demonstrated.
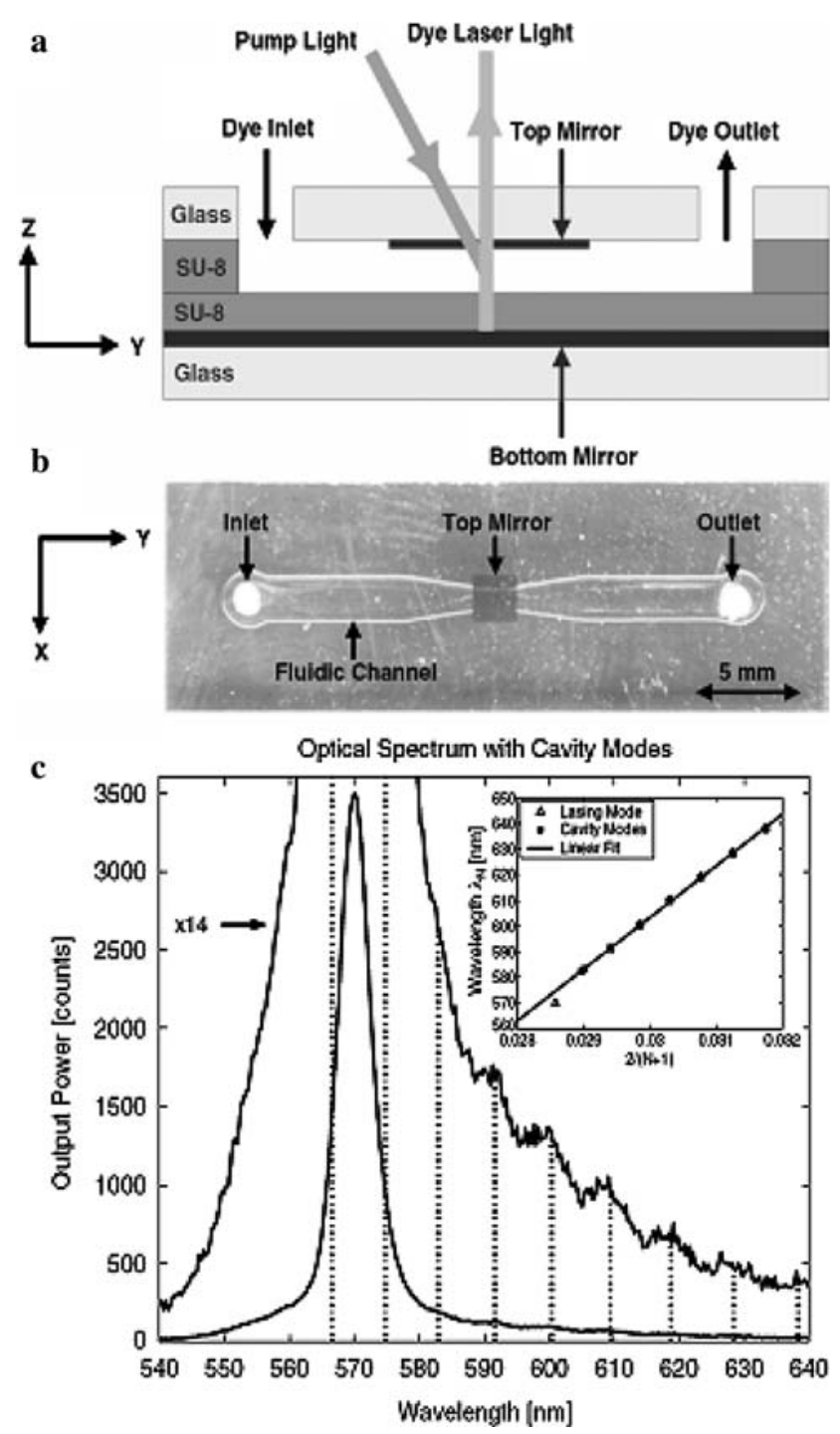

Fig. 6 The first on-chip optofluidic dye laser. a Schematic of the laser structure. b Topview optical micrograph of the device. c Lasing spectrum of the device. Small peaks marked by the dashed lines correspond to the longitudinal modes of the cavity. Reprinted with permission from Helbo et al. (2003) 


\subsection{Single-mode operation}

The early demonstrations of on-chip optofluidic dye lasers showed rather wide emission linewidths due to the multimode operation and low cavity qualities. Later, more efficient cavities were achieved using Bragg gratings, which enabled narrow linewidth single frequency output. Balslev and Kristensen (2005) demonstrated a single-mode dye laser on the basis of a multimode waveguide structure and a $\sim$ 130th Bragg grating. Approximately single spatial mode operation was observed due to the high losses of higher order spatial modes in the antiguiding segments.

A true single mode optofluidic dye laser was achieved using a single transverse mode liquid core waveguide embedded with a phase shifted 15th order distributed feedback (DFB) structure (Li et al. 2006a, b). Figure 7 shows the details of the device. The laser was fabricated on a monolithic PDMS chip using replica molding soft lithography (Xia and Whitesides 1998). A $1 \mathrm{mM}$ solution of Rhodamine 6G in a methanol and ethylene glycol mixture with the proper index $n=1.409$ was used to ensure single transverse mode operation. A 15th order Bragg grating (period $3 \mu \mathrm{m}$, length $8 \mathrm{~mm}$ ) gave rise to a FSR of $\sim 90 \mathrm{~nm}$, larger than the gain spectrum width. An effective $\pi / 2$ defect at the center of the grating ensured single frequency operation. When pumped with $6 \mathrm{~ns} 532 \mathrm{~nm}$ laser pulses, laser emission at $567.3 \mathrm{~nm}$ with a linewidth of $0.21 \mathrm{~nm}$ was obtained above the threshold of $0.8 \mathrm{~mJ} / \mathrm{cm}^{2}$.

The use of higher order Bragg gratings resulted in large device lengths and extra losses due to the out-of-plane scattering (Streifer et al. 1976). Therefore, considerable efforts have been made to achieve low-order optofluidic DFB dye lasers (Gersborg-Hansen and Kristensen 2006; Peroz et al. 2006; Li and Psaltis 2007). Figure 8 shows an optofluidic third order DFB dye laser (Gersborg-Hansen and Kristensen 2006). The alternating SU8 and dye solution formed the third-order Bragg grating. The $300 \mathrm{~nm}$ high nanofluidic channels were filled with the dye solution by capillary forces. Low threshold single frequency lasing $\left(10 \mu \mathrm{J} / \mathrm{mm}^{2}\right)$ was achieved with narrow linewidths $(<0.15 \mathrm{~nm})$.

\subsection{Tunable and multiple color output}

Shortly after the demonstration of the first optofluidic dye laser, tunable output was obtained by index and concentration tuning (Bilenberg et al. 2003; Galas et al. 2005; Gersborg-Hansen and Kristensen 2007). An ultrawide mechanical tuning range was achieved with the soft PDMS-based optofluidic DFB dye lasers (Li et al. 2006a, b). The higher order gratings allowed multiple color lasing from a single cavity when used with different dye
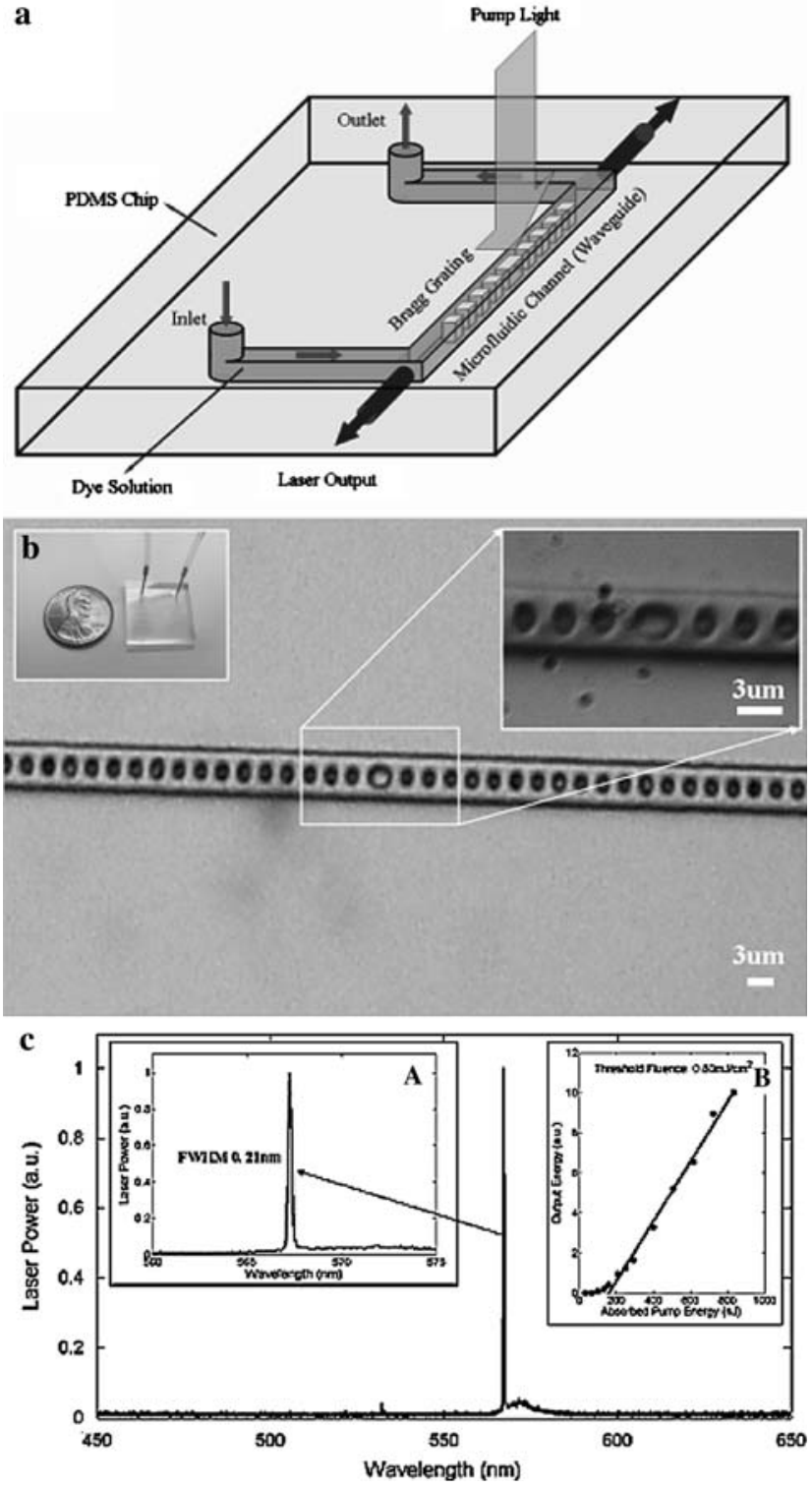

Fig. 7 Monolithic single mode optofluidic DFB dye laser in PDMS. a Schematic of the laser structure. A Bragg grating located in a liquid core optical waveguide formed the laser cavity. b Optical micrograph of the DFB laser cavity. A phase shifted 15th order Bragg grating was used. c Typical single mode lasing spectrum. Inset Light output versus light input curve. Threshold was about $0.8 \mathrm{~mJ} / \mathrm{cm}^{2}$. The laser output was at $567.3 \mathrm{~nm}$ with a linewidth of $0.21 \mathrm{~nm}$. Reprint with permission from Li et al. (2006a)

molecules. Due to the small Young's modulus of PDMS, very small forces were needed to change the grating period by stretching or compressing the laser chip. A nearly $60 \mathrm{~nm}$ tuning range was demonstrated from a single dye laser chip by combining two common dye molecules Rhodamine 6G and Rhodamine 101. An integrated array of five DFB dye lasers was also demonstrated as shown in Fig. 9. The parallel operation of such laser arrays should be of great interest for on-chip multiplexed sensing and 

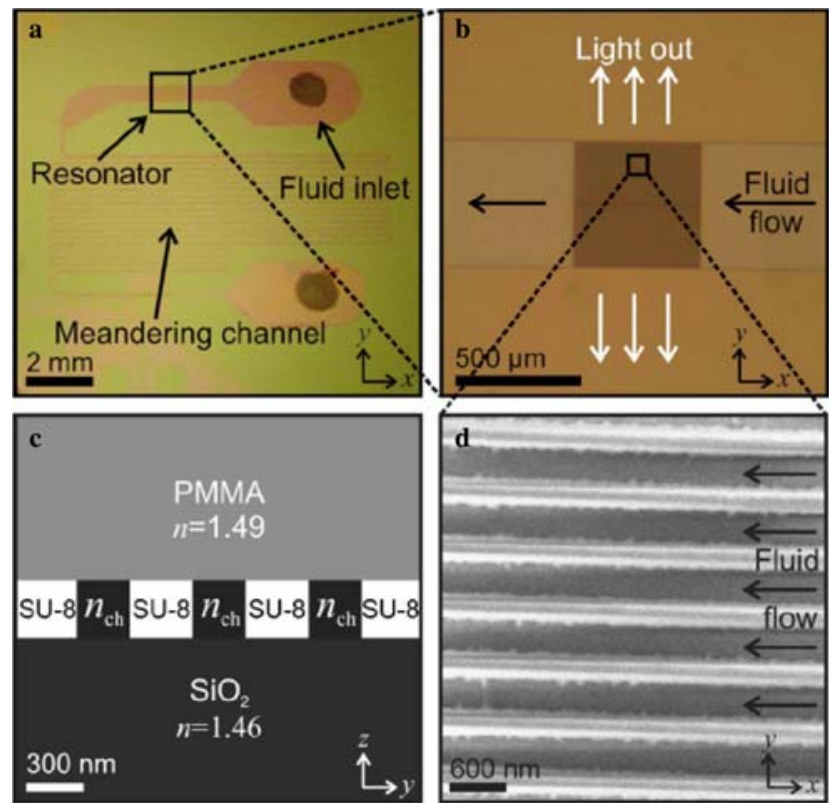

Fig. 8 Optofluidic third-order DFB dye laser. a Topview picture of the overall layout of the fabricated chip. The shallow $300 \mathrm{~nm}$ high meandering channel facilitates capillary filling of the laser resonator. b Optical micrograph of the DFB laser resonator embedded in the shallow meandering channel. c Side view sketch showing the layers of the DFB laser resonator. d Scanning electron micrograph showing the third-order Bragg grating of nanofluidic channels which constitutes the DFB laser resonator. Reprint with permission from GersborgHansen and Kristensen (2006)

spectroscopy applications. Index tuning of a fifth-order DFB dye laser was also achieved with an integrated microfluidic mixer (Li and Psaltis 2007).

By using a mixture of two dye molecules in a single solvent, dual-color output was achieve with an optical fiber-based Fabry-Perot cavity microfluidic dye laser (Kou et al. 2006). As shown in Fig. 10, two gold-coated cleaved optical fibers were placed in the PDMS channel to form a short Fabry-Perot cavity $(140 \mu \mathrm{m}$ cavity length). Collinear yellow $(559 \mathrm{~nm})$ and red lasing $(597 \mathrm{~nm})$ was obtained when a mixture of Rhodamine 6G and Surforhodamine 101 in ethanol was pumped by pulsed $532 \mathrm{~nm}$ laser light.

\subsection{Integration with other "lab-on-a-chip" functions}

The integration with other "lab-on-a-chip" components to build complete systems is a major motivation for building optofluidic dye lasers. However, very few demonstrations along this line have appeared so far. Galas et al. (2005) and $\mathrm{Li}$ et al. (2007) have demonstrated the integration of PDMS-based soft microfluidics with optofluidic dye lasers to change the laser properties. Such integration can also be used to build sensing and spectrometer devices. A device

\section{a Period}

$3080 \mathrm{~nm} 00000000000000000000000000000$

$3 0 4 0 \mathrm { nm } \longdiv { 0 0 0 0 0 0 0 0 0 0 0 0 0 0 0 0 0 0 0 0 0 0 0 0 0 0 0 0 0 0 0 }$

$3000 \mathrm{~nm} 0000000000000000000000000000$

$2640 \mathrm{~nm} 1000000000000000000000000000000006$

$2240 \mathrm{~nm} 2000000000000000000000000000000000000000$

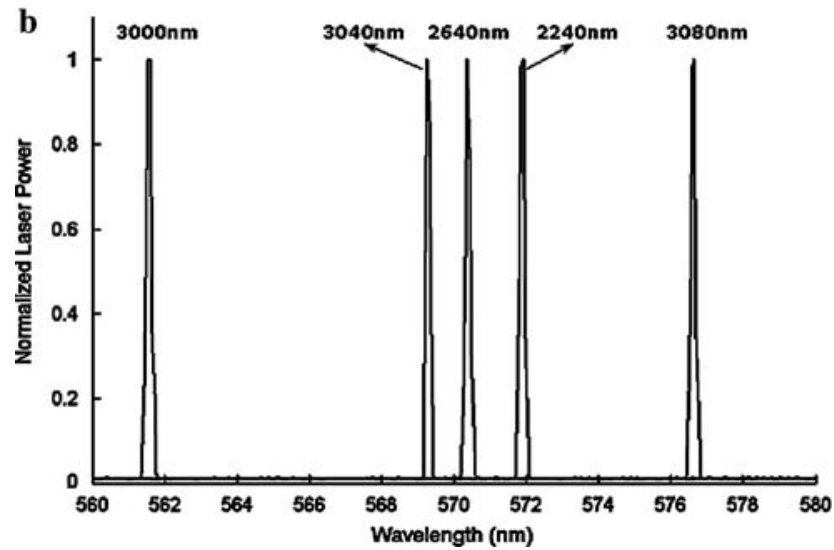

Fig. 9 An integrated optofluidic DFB dye laser array. a Optical micrograph of an integrated array of five optofluidic DFB dye lasers in PDMS. The grating period of each laser is given on the left. b Normalized laser output of the array using Rhodamine 6G dye solution as the gain medium. Reprinted with permission from Li et al. (2006b)

capable of performing highly sensitive intracavity absorption measurements on nanoliter samples was shown using a similar structure as shown in Fig. 10 (Galas et al. 2006). Concentrations of methylene blue as low as $10^{-6} \mathrm{~mol} / \mathrm{l}$ were detected.

Figure 11 shows a more sophisticated "lab-on-a-chip" system, which contained a liquid dye laser, optical waveguides, a microfluidic mixer, a sample cuvette and photodiodes (Balslev et al. 2006). The dye laser light at $576 \mathrm{~nm}$ was directly coupled into five waveguides that delivered the light to five different locations along a fluidic channel (cuvette) for absorbance measurements. The transmitted light was collected at the other side of the cuvette, again by waveguides, and finally detected by the photodiodes. Electrical read-out was accomplished by integrated metal connectors. To demonstrate the concerted operation of the integrated components, the authors performed an absorbance measurement on two different concentrations of xylenol orange dye. The photodiode signal showed two different values for concentrations of 0.06 and $0.12 \mathrm{mM}$. Despite the high level integration, this 

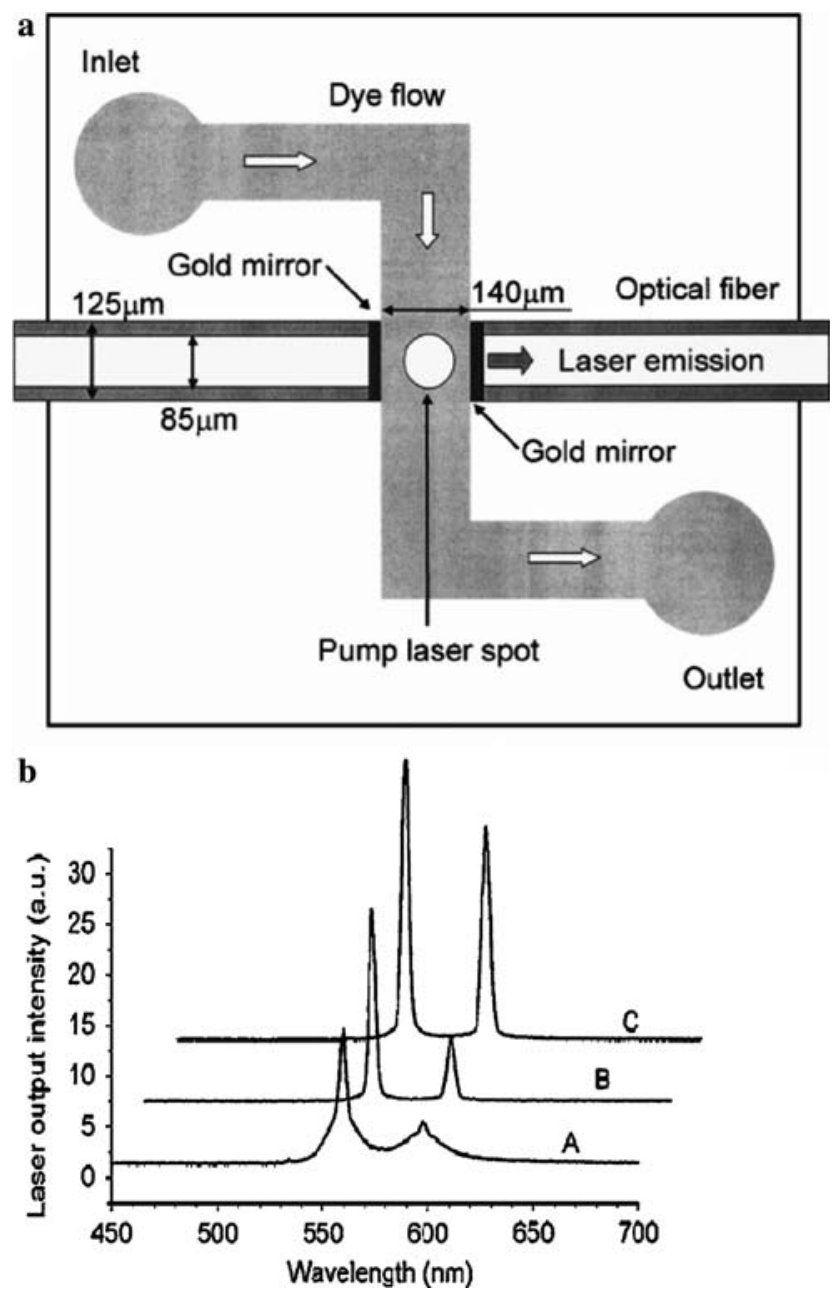

Fig. 10 Collinear dual-color laser emission from a microfluidic dye laser. a Schematic of the laser cavity and dye circulation channel. b Dual-laser spectra from the dye mixture under different experimental conditions. Curves $A$ to $C$ represent the laser lines at a flow rate of $2 \mu \mathrm{l} / \mathrm{min}$ and pump energy densities of $2.6,3.5$, and $4.5 \mu \mathrm{J} / \mathrm{mm}^{2}$, respectively. The lasing wavelengths were 559 and $597 \mathrm{~nm}$. Reprinted with permission from Kou et al. (2006)

device was still not a stand alone system due to the off chip optical pump and pressure source for driving the liquid flow.

\section{Future directions}

In order to fulfill the promise of optofluidic dye lasers as on-chip coherent light sources, the most important next step will be to integrate the optical pump source onto the same laser chip. On the basis of the lowest demonstrated laser threshold so far, this should be achievable with commercially available high power laser diodes ( $\mathrm{Li}$ and Psaltis 2007). Further improvements on the cavity $Q$ factor
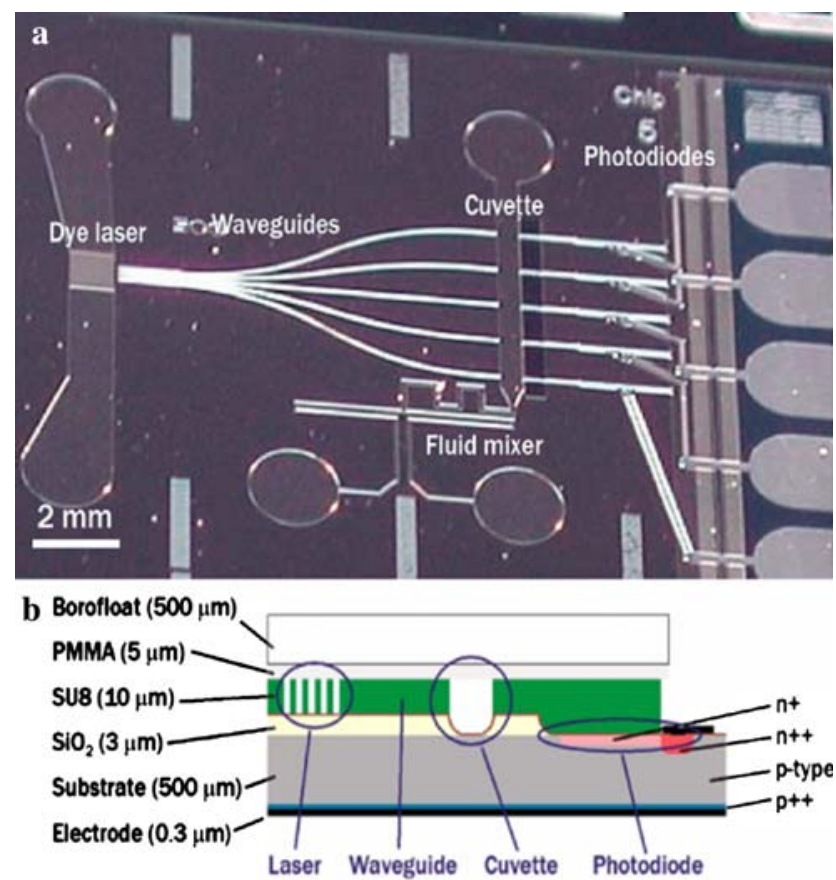

Fig. 11 Lab-on-a-chip system with integrated optical transducers. a Photograph of the lab-on-chip device with integrated microfluidic dye laser, optical waveguides, microfluidic mixer, sample cuvette and photodiodes. The chip footprint is $15 \mathrm{~mm} \times 20 \mathrm{~mm}$. b Cross section of the lab-on-a-chip device. Photodiodes are embedded in the silicon substrate, while the optical waveguides and the microfluidic network are defined in the SU8 film. The channels are sealed off by a borofloat glass lid bonded to the SU8 by means of a thin PMMA film. Reprinted with permission from Balslev et al. (2006)

and pump efficiency should also be studied to facilitate this integration.

One obvious direction is to follow the historical line of the conventional dye laser development. Compared with the best performances of conventional dye lasers (Schafer 1990), it is clear that optofluidic dye lasers still have a long way to go. Thus far, no CW operation or ultrashort pulse generation has been demonstrated. These are challenging tasks due to the small fluidic confinement in microfluidic devices. It seems the realization of cavity structures compatible with fast liquid flow will be a milestone for the future advances along this line.

Another direction that deserves special note is to develop other optofluidic microcavity structures, such as microrings (Hohimer et al. 1991), microdisks (McCall et al. 1992), microdroplets (Tzeng et al. 1984), circular Bragg gratings (Erdogan and Hall 1990) and photonic crystal microcavities (Painter et al. 1999). The potential high $Q$, small mode volume and the adaptive nature of such devices are very attractive for both fundamental studies of light-matter interactions and practical applications such as ultrasensitive biological and chemical sensing. 
Acknowledgments The authors gratefully acknowledge Axel Scherer, Zhaoyu Zhang, Teresa Emery and David Erickson for helpful discussions and their collaboration on this project. This research was supported by the DARPA center for optofluidic integration.

\section{References}

An KW (2001) Cylindrical and spherical microcavity lasers based on evanescent-wave-coupled gain. J Chin Chem Soc 48(3):461-468

An KW, Moon HJ (2003) Laser oscillations with pumping-independent ultrahigh cavity quality factors in evanescent-wavecoupled-gain microsphere dye lasers. J Phys Soc Jpn 72(4): $773-776$

Anna SL, Bontoux N, Stone HA (2003) Formation of dispersions using "flow focusing" in microchannels. Appl Phys Lett 82:364366

Azzouz H, Alkhafadiji L, Balslev S, Johansson J, Mortensen NA, Nilsson A, Kristensen A (2006) Levitated droplet dye laser. Opt Express 14(10):4374-4379

Balslev S, Kristensen A (2005) Microfluidic single mode laser using high order Bragg grating and antiguiding segments. Opt Express 13(1):344-351. http://wwwopticsinfobaseorg/abstractcfm?URI= oe131344

Balslev S, Jorgensen AM, Bilenberg B, Mogensen KB, Snakenborg D, Geschke O, Kutter JP, Kristensen A (2006) Lab-on-a-chip with integrated optical transducers. Lab Chip 6:213-217

Barnes MD, Whitten WB, Arnold S, Ramsey JM (1992) Homogeneous linewidths of Rhodamine-6G at room-temperature from cavity-enhanced spontaneous emission rates. J Chem Phys 97(10):7842-7845

Barnes MD, Ng KC, Whitten WB, Ramsey JM (1993) Detection of single Rhodamine-6 g molecules in levitated microdroplets. Anal Chem 65(17):2360-2365

Barnes MD, Kung CY, Whitten WB, Ramsey JM, Arnold S, Holler S (1996) Fluorescence of oriented molecules in a microcavity. Phys Rev Lett 76(21):3931-3934

Bilenberg B, Helbo B, Kutter JP, Kristensen A (2003) Tunable microfluidic dye laser. In: Proceedings of the 12th international conference on solid-state sensors, actuators and microsystems. Transducers, pp 206-209

Campillo AJ, Eversole JD, Lin HB (1991) Cavity quantum electrodynamic enhancement of stimulated-emission in microdroplets. Phys Rev Lett 67(4):437-440

Chabinyc ML, Chiu DT, Mcdonald JC, Stroock AD, Christian JF, Karger AM, Whitesides GM (2001) An integrated fluorescence detection system in poly(dimethylsiloxane) for microfluidic applications. Anal Chem 73(18):4491-4498

Chang RK, Campillo AJ (eds) (1996) Optical processes in microcavities, World Scientific, Singapore

Cheng Y, Sugioka K, Midorikawa K (2004) Microfluidic laser embedded in glass by three-dimensional femtosecond laser microprocessing. Opt Lett 29(17):2007-2009

Choi YS, Moon HJ, An KY, Lee SB, Lee JH, Chang JS (2001) Ultrahigh- $Q$ microsphere dye laser based on evanescent-wave coupling. J Korean Phys Soc 39(5):928-931

Coldren LA, Corzine SW (1995) Diodelasers and photonic integrated circuits, Wiley-Interscience, New York

Davis CC (1996) Lasers and electro-optics. Cambridge University Press, Cambridge

De Martini F, Jacobovitz GR (1988) Anomalous spontaneousstimulated-decay phase transition and zero-threshold laser action in a microscopic cavity. Phys Rev Lett 60(17):1711-1714

Duarte FJ, Hillman LW (eds) (1990) Dye laser principles, Academic, New York
Duarte FJ (eds) (1992) Selected papers on dye lasers, SPIE milestone series 45. SPIE Optical Engineering Press, Bellingham

Erdogan T, Hall DG (1990) Circularly symmetrical distributed feedback semiconductor-laser: an analysis. J Appl Phys 68(4): 1435-1444

Erickson D, Li DQ (2004) Integrated microfluidic devices. Anal Chim Acta 507:11-26

Eversole JD, Lin HB, Huston AL, Campillo AJ, Leung PT, Liu SY, Young K (1993) High-precision identification of morphologydependent resonances in optical processes in microdroplets. J Opt Soc Am B 10:1955-1968

Fujiwara H, Sasaki K (1999) Lasing of a microsphere in dye solution. Jpn J Appl Phys 38(9a):5101-5104

Galas JC, Torres J, Belotti M, Kou Q, Chen Y (2005) Microfluidic tunable dye laser with integrated mixer and ring resonator. Appl Phys Lett 86(26):264101

Galas JC, Peroz C, Kou Q, Chen Y (2006) Microfluidic dye laser intracavity absorption. Appl Phys Lett 89(22):224101

Gersborg-Hansen M, Kristensen A (2006) Optofluidic third order distributed feedback dye laser. Appl Phys Lett 89:103518

Gersborg-Hansen M, Kristensen A (2007) Tunability of optofluidic distributed feedback dye lasers. Opt Express 15:137-142

Hansch TW (2005) Edible lasers and other delights from the 1970's. Opt Photon News 16:14-16

Helbo B, Kristensen A, Menon A (2003) A micro-cavity fluidic dye laser. J Micromech Microeng 13(2):307-311

Hohimer JP, Craft DC, Hadley GR, Vawter GA, Warren ME (1991) Single-frequency continuous-wave operation of ring resonator diode lasers. Appl Phys Lett 59:3360-3362

Hong JW, Quake SR (2003) Integrated nanoliter systems. Nat Biotechnol 21:1179-1183

Hunsperger RG (2002) Integrated optics, 5th edn. Springer, New York

Ippen EP, Shank CV, Dienes A (1971) Rapid photobleaching of organic laser dyes in continuously operated devices. J Quantum Electron 7(4):178-179

Ippen EP, Shank CV (1972) Evanescent field-pumped dye laser. Appl Phys Lett 21(7):301-303

Johnston Jr TF (1987) Tunable dye lasers, encyclopedia of physical science and technology, vol 14. Academic, New York, pp 96-141

Kazes M, Lewis DY, Ebenstein Y, Mokari T, Banin U (2002) Lasing from semiconductor quantum rods in a cylindrical microcavity. Adv Mater 14(4):317

Kou Q, Yesilyurt I, Chen Y (2006) Collinear dual-color laser emission from a microfluidic dye laser. Appl Phys Lett 88(9):091101

Lermer N, Barnes MD, Kung CY, Whitten WB, Ramsey JM (1997) High efficiency molecular counting in solution: single-molecule detection in electrodynamically focused microdroplet streams. Anal Chem 69(11):2115-2121

Li ZY, Zhang ZY, Emery T, Scherer A, Psaltis D (2006a) Single mode optofluidic distributed feedback dye laser. Opt Express 14(2):696-701. http://www.opticsinfobase.org/abstract.cfm? URI=oe-14-2-696

Li ZY, Zhang ZY, Scherer A, Psaltis D (2006b) Mechanically tunable optofluidic distributed feedback dye laser. Opt Express 14(22):10494-10499. http://wwwopticsinfobaseorg/abstractcfm? URI $=$ oe 142210494

Li ZY, Psaltis D (2007) Optofluidic distributed feedback dye lasers. IEEE J Sel Topics Quantum Electron 13(2):185-193

Lin HB, Huston AL, Justus BL, Campillo AJ (1986) Some characteristics of a droplet whispering-gallery-mode laser. Opt Lett 11(10):614-616

Lin HB, Eversole JD, Campillo AJ (1990) Vibrating orifice droplet generator for precision optical studies. Rev Sci Instrum 61(3):1018-1023 
Lin HB, Eversole JD, Campillo AJ (1992a) Spectral properties of lasing microdroplets. J Opt Soc Am B 9:43-50

Lin HB, Eversole JD, Merritt CD, Campillo AJ (1992b) Cavity modified spontaneous-emission rates in liquid microdroplets. Phys Rev A 45:6756-6760

Maune B, Loncar M, Witzens J, Scherer A (2004) Liquid-crystal electric tuning of a photonic crystal laser. Appl Phys Lett 85(3):360-362

McCall SL, Levi AF, Slusher RE, Pearton SJ, Logan RA (1992) Whispering-gallery mode microdisk lasers. Appl Phys Lett 60:289-291

Mekis A, Noeckel JU, Chen G, Stone AD, Chang RK (1995) Ray chaos and $Q$ spoiling in lasing droplets. Phys Rev Lett 75:26822685

Monat C, Domachuk P, Eggleton BJ (2007) Integrated optofluidics: a new river of light. Nat Photonics 1:106-114

Moon HJ, Chough YT, Kim JB, An KW (2000a) Cavity- $Q$-driven spectral shift in a cylindrical whispering-gallery-mode microcavity laser. Appl Phys Lett 76(25):3679-3681

Moon HJ, Chough YT, An KW (2000b) Cylindrical microcavity laser based on the evanescent-wave-coupled gain. Phys Rev Lett 85(15):3161-3164

Nockel JU, Stone AD, Chen G, Grossman HL, Chang RK (1996) Directional emission from asymmetric resonant cavities. Opt Lett 21:1609-1611

Painter O, Lee RK, Scherer A, Yariv A, O’Brien JD, Dapkus PD, Kim I (1999) Two-dimensional photonic band-gap defect mode laser. Science 284(5421):1819-1821

Pendock G, Mackenzie HS, Payne FP (1992) Tapered optical fibre dye laser. Electron Lett 28:149-150

Pendock GJ, Mackenzie HS, Payne FP (1993) Dye-lasers using tapered optical fibers. Appl Opt 32(27):5236-5242

Periasamy N, Bor Z (1981) Distributed feedback laser action in an optical fiber by evanescent field coupling. Opt Commun 39(5):298-302

Periasamy N, Schafer FP (1981) Laser amplification in an optical fiber by evanescent field coupling. Appl Phys 24(3):201-203

Peroz C, Galas JC, Le Gratiet L, Chen Y, Shi J (2006) Compact dye laser on a chip fabricated by ultraviolet nanoimprint lithography. Appl Phys Lett 89(24):243109

Peterson OG (1979) Dye lasers, methods of experimental physics, vol 15A. Academic, New York, pp 251-359

Peterson OG, Webb JP, Mcclolgin WC (1971) Organic dye laser threshold. J Appl Phys 42(5):1917-1928

Psaltis D, Quake SR, Yang CH (2006) Developing optofluidic technology through the fusion of microfluidics and optics. Nature 442:381-386

Purcell EM (1946) Spontaneous emission probabilities at radio frequencies. Phys Rev 69:681-681

Qian SX, Snow JB, Tzeng HM, Chang RK (1986) Lasing droplets: highlighting the liquid-air interface by laser emission. Science 231(4737):486-488

Russell P (2003) Photonic crystal fibers. Science 299:358-362

Schafer FP (eds) (1990) Dye lasers: topics in applied physics 1, 3rd edn. Springer, Heidelberg

Shank CV (1975) Physics of dye lasers. Rev Mod Phys 47(3):649657

Snavely BB (1969) Flashlamp-excited organic dye lasers. Proc IEEE 57(8):1374-1390
Shopova SI, Zhu HY, Fan XD, Zhang P (2007) Optofluidic ring resonator based dye laser. Appl Phys Lett 90:221101

Silfvast WT (2004) Laser fundamentals, 2nd edn. Cambridge University Press, Cambridge

Sorokin PP, Lankard JR, Moruzzi VL, Hammond EC (1968) Flashlamp-pumped organic-dye lasers. J Chem Phys 48(10): 4726-4741

Streifer W, Burnham RD, Scifres DR (1976) Radiation losses in distributed feedback lasers and longitudinal mode selection. IEEE J Quantum Electron 12(11):737-739

Suzuki M, Yokoyama H, Brorson SD, Ippen EP (1991) Observation of spontaneous emission lifetime change Of dye-containing Langmuir-Blodgett films in optical microcavities. Appl Phys Lett 58(10):998-1000

Svelto O (1998) Principles of lasers. 4th edn. Plenum, New York

Thorsen T, Roberts RW, Arnold FH, Quake SR (2001) Dynamic pattern formation in a vesicle-generating microfluidic device. Phys Rev Lett 86:4163-4166

Thorsen T, Maerkl SJ, Quake SR (2002) Microfluidic large-scale integration. Science 298:580-584

Tona M, Kimura M (2000) Novel lasing modes observed in a levitated single dye-doped microdroplet. J Phys Soc Jpn 69(11):3533-3535

Tzeng HM, Wall KF, Long MB (1984) Evaporation and condensation rates of liquid droplets deduced from structure resonances in the fluorescence spectra. Opt Lett 9:273-275

Unger MA, Chou HP, Thorsen T, Scherer A, Quake SR (2000) Monolithic microfabricated valves and pumps by multilayer soft lithography. Science 288:113-116

Vahala KJ (2003) Optical microcavities. Nature 424:839-846

Vasdekis AE, Town GE, Turnbull GA, Samuel IDW (2007) Fluidic fibre dye lasers. Opt Express 15:3962-3967. http://www. opticsinfobase.org/abstract.cfm?URI=oe-15-7-3962

Verpoorte E (2003) Chip vision: optics for microchips. Lab Chip $3(3): 42 \mathrm{~N}-52 \mathrm{~N}$

Vezenov DV, Mayers BT, Conroy RS, Whitesides GM, Snee PT, Chan Y, Nocera DG, Bawendi MG (2005) A low-threshold, high-efficiency microfluidic waveguide laser. J Am Chem Soc 127(25):8952-8953

Whitesides GM (2006) The origins and the future of microfluidics. Nature 442:368-373

Wolfe DB, Conroy RS, Garstecki P, Mayers BT, Fischbach MA, Paul KE, Prentiss M, Whitesides GM (2004) Dynamic control of liquid-core/liquid-cladding optical waveguides. Proc Natl Acad Sci USA 101(34):12434-12438

Xia YN, Whitesides GM (1998) Soft lithography. Annu Rev Mater Sci 28:153-184

Yang J, Guo LJ (2006) Optical sensors based on active microcavities. IEEE J Sel Topics Quantum Electron 12(1):143-147

Yokoyama H (1992) Physics and device applications of optical microcavities. Science 256(5053):66-70

Yokoyama H, Brorson SD (1989) Rate equation analysis of microcavity lasers. J Appl Phys 66(10):4801-4805

Yokoyama H, Suzuki M, Nambu Y (1991) Spontaneous emission and laser oscillation properties of microcavities containing a dye solution. Appl Phys Lett 58(23):2598-2600

Zeidler G (1971) Optical waveguide technique with organic dye lasers. J Appl Phys 42(2):884-886 